Classification

Physics Abstracts

$21.60-21.60 \mathrm{E}$

\title{
On the structure of ${ }^{225} \mathrm{Ra}$
}

\author{
R. Piepenbring \\ Institut des Sciences Nucléaires, 53, avenue des Martyrs, 38026 Grenoble Cedex, France
}

(Reçu le 16 juillet 1984, accepté le 7 septembre 1984)

\begin{abstract}
Résumé. - Les arguments habituellement avancés pour affirmer que le ${ }^{225} \mathrm{Ra}$ a une déformation octupolaire stable sont discutés en détail. Beaucoup apparaissent comme nécessaires mais non suffisants et sont donc à utiliser avec prudence.
\end{abstract}

\begin{abstract}
Various arguments are usually invoked to favour stable octupole deformation for ${ }^{225} \mathrm{Ra}$. A detailed discussion of these arguments is given. Most of them are necessary but not sufficient criteria for stable octupole deformation, and must therefore be used with caution.
\end{abstract}

It is well known that the octupole correlations play an important role in the region of $\mathrm{Ra}$ and $\mathrm{Th}$. A deformation of $\mathrm{Y}_{30}$ type is needed to understand the low lying level structure of these nuclei. It is however not yet completely clear if the octupole degree of freedom leads to a stable deformation or to vibrations. A detailed experimental study of ${ }^{225} \mathrm{Ra}$ is now available [1], whose results have been analysed in two recent letters $[2,3]$ and in a more elaborated article by Leander and Sheline [4]. The last three papers show some evidence for stable octupole deformation in ${ }^{225} \mathrm{Ra}$. Various arguments are given in references [2-4] in favour of this interpretation. The aim of the present Letter is to discuss these arguments in detail and to show that, at least, some of those appear to be not sufficient criteria for octupole deformation.

Let us first list the different arguments of the authors of references [2-4].

a) It turned out to be difficult to get a consistent description of the different rotational bands within the Nilsson model assuming no stable octupole deformation $[2,4]$.

b) The fact that the two $K=1 / 2^{ \pm}$bands have decoupling parameters $a$ of nearly same absolute values but with opposite signs is another criterion for stable octupole deformation [3].

c) The presence of two rotational bands $K=1 / 2$ and $K=3 / 2$ with nearly degenerate parity doublets is one of the most simple criteria for stable octupole deformation in odd- $A$ nuclei [3].

d) Despite extensive experimental studies of references $[5,6]$, one has not found any vibrational two-phonon octupole vibrational $0^{+}$state in ${ }^{222,224} \mathrm{Ra}$ at around double the energy of the onephonon state $[2,3]$.

We shall now analyse each of these points in detail. Five rotational bands are observed [1] with band head energies lower than $250 \mathrm{keV}$. One of these has clearly been interpreted as built on the $5 / 2+633$ Nilsson state. The usual Nilsson assignments for the four other bands with $K=1 / 2^{ \pm}$and $K=3 / 2^{ \pm}$are less evident. Two well known couplings are expected to play here an important role. First, some of the single neutron levels, expected in this mass region for small quadrupole deformation, arise from the spherical orbits $j_{15 / 2}$ and $i_{11 / 2}$ having large $j$ values and Article published online by EDP Sciences and available at http://dx.doi.org/10.1051/jphyslet:0198400450210102300 
hence, leading to strong Coriolis coupling between the deformed states. This coupling has not been explicitly introduced in [2-4]. Second, the presence of a low lying octupole state in the even isotopes induces a strong coupling of the odd-neutron to the octupole mode. This coupling is just the challenging problem we deal with.

To describe the experimental data, one may use a priori a model either with a stable octupole deformation (Fig. 1a) or with reflection symmetry (Fig. 1b). In the first case the potential energy curve has two minima located at a deformation $\pm \varepsilon_{3} \neq 0$. The difference between the stable (1a) and unstable (1b) deformation depends on the height of the barrier at $\varepsilon_{3}=0$ in figure $1 \mathrm{a}$. The level order $0^{+}, 2^{+}, 1^{-}, 4^{+}, 3^{-}$observed in ${ }^{224} \mathrm{Ra}$ and the level order $3 / 2^{+}, 5 / 2^{+}, 7 / 2^{+}, 3 / 2^{-}$observed in ${ }^{225} \mathrm{Ra}$ suggest (see Ref. [7]) that this barrier is not very high. In that case the whole difference between the two versions (a) and (b) becomes not so essential and may even be reduced to using different representations. One may therefore use either a reflection asymmetric or symmetric basis. The basis which is most nearly diagonal will appear to be the most suited to give the best interpretation.

Let us here start with the symmetric assumption and look if a coherent description of the low energy spectrum given in reference [1] is still possible in the frame of the unified model of Bohr and Mottelson. In this approach, the odd-nucleus basic wavefunction still writes

$$
\Psi_{I M K}^{v}=\frac{(2 I+1)^{1 / 2}}{4 \pi}\left\{\mathfrak{D}_{M K}^{I} \phi_{v}+p \mathfrak{D}_{M-K}^{I} \phi_{-v}\right\}
$$

where $\phi_{v}$ represents now the intrinsic wavefunction taking into account the coupling of the single quasiparticle to the octupole vibration, and replaces the usual Nilsson wavefunction $\chi_{v}$. As usual, the Coriolis forces couple $\Psi_{I M K}^{v}$ states with $|\Delta K|=1$.

The phase factor $p$ takes a value depending on the definition of the conjugate state $\phi_{-v}$.

We test the coherence of the experimental level scheme by use of the effective matrix method, developed earlier [8] and based on the inversion of the eigenvalue problem.

For the negative parity levels, we restrict the basis (1) to two intrinsic states having $K=1 / 2$ and $K=3 / 2$. The problem has then 5 parameters : the intrinsic energies $\varepsilon_{1 / 2}$ and $\varepsilon_{3 / 2}$, the effective inertia parameter $A_{\text {eff }}=\frac{\hbar^{2}}{2 J}$, the effective Coriolis matrix element $C_{\text {eff }}=\left|\left\langle\phi_{3 / 2}\left|j_{+}\right| \phi_{1 / 2}\right\rangle\right|^{2}$

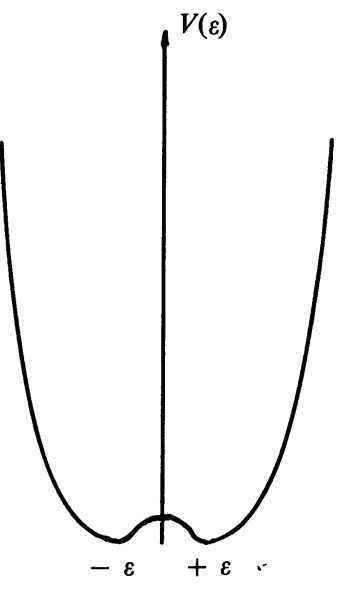

(a)

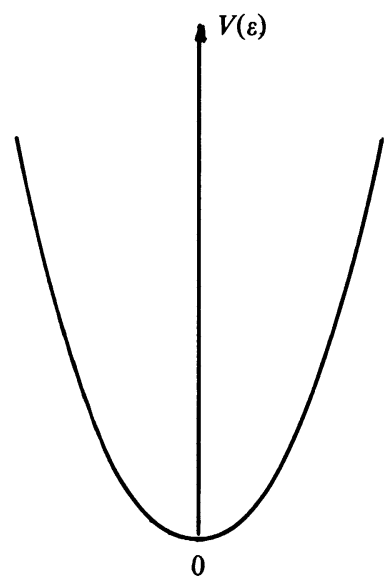

(b)

Fig. 1. - Potential energy curves for stable octupole deformation (part a) and for reflection symmetric deformation (part b). 
and the effective decoupling parameter $a_{\text {eff }}=-\left\langle\phi_{1 / 2}\left|j_{+}\right| \phi_{-1 / 2}\right\rangle$. It is very easy to fit these five parameters to the six observed energies (table I). The adjusted values of the physical parameters of interest here are :

$$
A_{\text {eff }}=5.87 \mathrm{keV} \quad C_{\text {eff }}=3.76 \quad a_{\text {eff }}=-2.22 .
$$

For the positive parity levels we can use either a basis (1) with two intrinsic states, $K=1 / 2$ and $K=3 / 2$, or one with three states adding the $K=5 / 2$ configuration. With the first choice we have eight levels for the same number of parameters (five) as in the negative parity case. With the second choice we have two additional parameters $C^{\prime}=\left|\left\langle\phi_{5 / 2}\left|j_{+}\right| \phi_{3 / 2}\right\rangle\right|^{2}$ and $\varepsilon_{5 / 2}$. We then end with seven parameters for thirteen levels. A satisfactory fit can be obtained in the two cases. The two-band coupling leads however to a better general agreement. We shall therefore restrict our discussion to this case (table II) where the adjusted values of the interesting parameters are

$$
A_{\text {eff }}=5.56 \mathrm{keV} \quad C_{\text {eff }}=1.16 \quad a_{\text {eff }}=1.30 \text {. }
$$

We may add that these values are somewhat sensitive to the number and/or spin values of the levels introduced in the fit. Giving or using too precise values of the effective inertia parameter or effective matrix elements of $j_{+}$has therefore no physical meaning. The importance of the Coriolis coupling can be seen by comparing the values of $A_{\text {eff }}$ and $a_{\text {eff }}$ obtained here in equations (2) and (3) with the values given in [3] (and [4] respectively)

$$
\begin{aligned}
& A_{\text {eff }}=4.937 \mathrm{keV} \\
& a_{\text {eff }}=-2.594(-2.4)
\end{aligned}
$$

for the negative parity band $1 / 2$ and

$$
\begin{aligned}
& A_{\text {eff }}=5.63 \mathrm{keV} \\
& a_{\text {eff }}=1.534(1.5)
\end{aligned}
$$

for the $K=1 / 2^{+}$band.

The results shown in tables I and II clearly demonstrate that one can obtain, within the collective model of Bohr and Mottelson, a coherent description for the low energy and low spin

Table I. - Observed and calculated energies $E(I)$ in $\mathrm{keV}$ of negative parity in ${ }^{225} \mathrm{Ra}$ for the two lowest rotational bands.

\begin{tabular}{|c|c|c|}
\hline$I$ & $E(I)_{\exp }$ & $E(I)_{\mathrm{cal}}$ \\
\hline $1 / 2$ & 55.2 & 55.2 \\
\hline $3 / 2$ & 31.6 & 31.7 \\
& 225.1 & 225.1 \\
\hline $5 / 2$ & 120.3 & 120.3 \\
& 260.2 & 260.2 \\
\hline $7 / 2$ & 69.4 & 69.4 \\
& & {$[302]$.} \\
\hline
\end{tabular}


Table II. - Same as in table I, but for positive parity.

\begin{tabular}{|c|c|c|}
\hline$I$ & $E(I)_{\exp }$ & $E(I)_{\text {cal }}$ \\
\hline $1 / 2$ & 0. & 0. \\
\hline $3 / 2$ & 42.8 & 37.5 \\
& 149.9 & 150.1 \\
\hline $5 / 2$ & 25.4 & 28.1 \\
& 179.9 & 178.9 \\
\hline $7 / 2$ & 111.7 & 114.5 \\
& 220. & 221.3 \\
\hline $9 / 2$ & 100.6 & 99.3 \\
& & {$[271]$.} \\
\hline
\end{tabular}

spectrum of ${ }^{225} \mathrm{Ra}$. The problem is now to find an interpretation of the empirical values given in (2) and (3). We first note that the values of the effective inertia parameters do not differ too much for the two sets of bands. Further they are typical of nuclei with small deformations of the considered transition region. The interpretation of the empirical values of the effective decoupling parameters is more delicate. In terms of pure Nilsson configurations this is of course a puzzling problem, as has been discussed in [2-4]. For the negative parity $K=1 / 2$ band, one expects the $1 / 2-770$ Nilsson orbit. For nuclei with small quadrupole deformation (say $\varepsilon_{2}=0.15$ ), the single particle decoupling factor $a_{\mathrm{sp}}$, calculated with the parameters of Lamm [9], is $a_{\mathrm{sp}} \simeq-7.8$ which has a much larger absolute value than the effective one $a_{\text {eff }}=-2.22$. For the $K=1 / 2^{+}$ band the situation is even more difficult. The two possible candidates for the Nilsson configuration are $1 / 2+631$ or $1 / 2+640$. With the usual parameters of [9] the first one lies too high in energy and the second too low. The same situation occurs if one uses [10] a Woods-Saxon potential with axial symmetry. It has been shown by Ragnarsson [2] that the introduction of an octupole deformation helps to bring the $1 / 2+631$ down in energy. The use of the sophisticated folded Yukawa single-particle potential [7] indicates the same necessity of an $\varepsilon_{3} \neq 0$, i.e. a reflection asymmetry, to get the $1 / 2+631$ down in energy. This appears of course as a strong evidence for stable octupole deformation. On the other hand, it is well known that in order to get the single particle relative energies exactly right in the calculation of odd- $A$ nuclear spectra, it is necessary to make some empirical adjustments, which may be even somewhat larger than the expected inaccuracy of the used single particle scheme [4, 11]. Earlier work by Rekstad and Lovhøiden [12] has also demonstrated that a re-examination of the parameters $\kappa$ and $\mu$ of the Nilsson potential for the transitional regions is necessary. We therefore consider the present argument as less strong and continue our discussion by assuming that a symmetric shape may still be used. The single particle decoupling factors of $1 / 2+631$ and $1 / 2+640$ with the parameters of [9] are 0.86 and -5.1 , respectively. These values have nothing in common with the empirical value 1.3. The situation gets even worse if one uses the parameters of Chi like in [3] or a Woods-Saxon potential [10] for which small negative values are obtained for $a_{\mathrm{sp}}(1 / 2+631)$. The situation becomes much better if one switches from the pure Nilsson functions $\chi_{v}$ to the intrinșic functions $\phi_{v}$. If one restricts to the particle-octupole $K=0^{-}$coupling 
one has :

$$
\phi_{v}=\left\{S_{v} C_{v}^{\dagger}+\sum_{v^{\prime}} S_{v^{\prime}} Q_{30}^{\dagger} C_{v^{\prime}}^{\dagger}+\sum_{v^{\prime \prime}} S_{v^{\prime \prime}}\left(Q_{30}^{\dagger}\right)^{2} C_{v^{\prime \prime}}^{\dagger}+\cdots\right\}|0\rangle
$$

where $Q_{30}^{\dagger}$ is the octupole phonon operator. The operators $C_{v}^{\dagger}, C_{v^{\prime}}^{\dagger}, C_{v^{\prime \prime}}^{\dagger}$ create quasiparticles with same $\Omega_{v}=\Omega_{v^{\prime}}=\Omega_{v^{\prime \prime}}=K$ values and adequate parities (e.g. in Eq. (4) $v^{\prime}$ has opposite parity of $v$ or $\left.v^{\prime \prime}\right)$. The coefficients $S_{v}, S_{v^{\prime}}, S_{v^{\prime \prime}}$ can be obtained in a microscopic way by using the multiphonon method applied to odd-mass nuclei [13]. The empirical values of the effective decoupling factors are then to be compared to the intrinsic decoupling parameters $a_{\text {int }}$ defined by

$$
a_{\text {int }}=-\left\langle\phi_{v=1 / 2}\left|j_{+}\right| \phi_{v=-1 / 2}\right\rangle \text {. }
$$

To push the discussion further, we shall make two other assumptions, which may appear too drastic, but which are made only to show that the argument (b) on the decoupling parameters is not sufficient. We first neglect $S_{v^{\prime \prime}}$ and higher multiphonon terms in (4). Then by taking properly into account the conjugation properties of $\phi_{v}$ we have shown [14] that

$$
a_{\mathrm{int}}=S_{v}^{2} a_{\mathrm{sp}}(v)+\sum_{\lambda \mu} S_{\lambda} S_{\mu}\left\langle\chi_{\lambda=1 / 2}\left|j_{+}\right| \chi_{\mu=-1 / 2}\right\rangle P
$$

where $P=u_{i} u_{\mu}+v_{\lambda} v_{\mu} \lesssim 1$ is a correction factor due to the pairing correlations. We simplify further the argument and assume that we can restrict ourselves to the two Nilsson orbitals $1 / 2-770$ and $1 / 2+631$. The latter choice is preferred to $1 / 2+640$ according to the transfer reaction results of [1]. In that over-simplified case, equation (4) writes :

$$
\begin{aligned}
& \phi_{1 / 2-}=\alpha C_{1 / 2-}^{\dagger}|0\rangle+\beta Q_{30}^{\dagger} C_{1 / 2+}^{\dagger}|0\rangle \\
& \phi_{1 / 2+}=\gamma C_{1 / 2+}^{\dagger}|0\rangle+\delta Q_{30}^{\dagger} C_{1 / 2-}^{\dagger}|0\rangle
\end{aligned}
$$

where

$$
\alpha^{2}+\beta^{2}=\gamma^{2}+\delta^{2}=1
$$

for negative and positive parity, respectively. The intrinsic decoupling parameters of equation (6) are then simply :

$$
\begin{aligned}
& a_{\mathrm{int}}\left(1 / 2^{-}\right)=\alpha^{2} a_{\mathrm{sp}}(1 / 2-770)-\beta^{2} a_{\mathrm{sp}}(1 / 2+631) \\
& a_{\mathrm{int}}\left(1 / 2^{+}\right)=\gamma^{2} a_{\mathrm{sp}}(1 / 2+631)-\delta^{2} a_{\mathrm{sp}}(1 / 2-770) .
\end{aligned}
$$

From equation (11) we immediately see that the negative value of $a_{\mathrm{sp}}(1 / 2-770)$ helps to enhance the too small positive or even negative $a_{\mathrm{sp}}(1 / 2+631)$ and to get a value comparable to $a_{\mathrm{int}}\left(1 / 2^{+}\right)$. On the other hand, in equation (10) the fact that $\alpha^{2}<1$ and the small value of $a_{\mathrm{sp}}(1 / 2+631)$ explain a lowering of the absolute value of $a_{\mathrm{int}}\left(1 / 2^{-}\right)$compared to that of $a_{\mathrm{sp}}(1 / 2-770)$. One could continue the game. By choosing single particle values for $a_{\mathrm{sp}}$, by using the empirical values for $a_{\text {int }}\left(1 / 2^{ \pm}\right)$and the normalization condition (9), one could deduce the mixing coefficients $\alpha$, $\beta, \gamma, \delta$ of the wavefunctions (7) and (8). But, according to the too drastic assumptions made, this would not have a real physical meaning. We simply note that, by using the $a_{\mathrm{sp}}$ values indicated in [3], one remains with plausible values

$$
\alpha=0.59 \quad \beta=0.81 \quad \gamma=0.88 \quad \delta=0.48
$$

for the mixing coefficients. We checked that these values also allow a description of the magnetic moment $\mu$ with reasonable values of the gyromagnetic factors $g_{\mathrm{s}}$ and $g_{\mathrm{R}}$, as well as an explanation of the empirical values of the $C_{\text {eff }}$ where the pairing corrections have some small effects. Cri- 
terion (b) that the decoupling parameters for $K=1 / 2^{ \pm}$have opposite values, used in [3] as a criterion for stable octupole deformation, is certainly necessary but not at all sufficient as we have just showed. Besides, this has been recognized later on by the same authors in references $[1,4]$. It has also been claimed [4] that further evidence for parity mixed single particle states (i.e. reflection asymmetry) might be found in a deviation of the decoupling factor from experimental systematics. We feel that even this statement should be used with caution for the following reasons : it has been found that the calculated decoupling factor $a\left(1 / 2^{-}\right)$for $\varepsilon_{3} \neq 0$ is a rapidly decreasing function of $\varepsilon_{3}$. A slight deformation change from one nucleus to another may therefore explain strong changes in the systematics. The same fact can as well be explained within the reflection symmetry assumption. A slight change of the energy of the octupole vibrational state from one nucleus to another changes the mixing coefficients $\alpha$ and $\beta$, and, according to the large value of the $\left|a_{\mathrm{sp}}(1 / 2-770)\right|$ also the calculated $a_{\mathrm{int}}\left(1 / 2^{-}\right)$.

Let us come to criterion (c), which claims that the presence of two rotational bands $K=1 / 2$ and $K=3 / 2$ with nearly degenerate parity doublets is one of the simplest ones for stable octupole deformation in odd- $A$ nuclei. Here too, one can find some arguments against this assertion. If this reasoning works for $K=1 / 2$ and $K=3 / 2$, it should also work for $K=5 / 2$, and ask for a $5 / 2^{-}$band in the proximity of the observed $5 / 2^{+}$at $236 \mathrm{keV}$. Such a finding has not been reported in [1] where the first possible candidate appears at $1225 \mathrm{keV}$. One could as well say that opposite parity states in odd- $A$ nuclei can be attributed to unrelated Nilsson orbitals. This is especially the case when two spherical subshells of opposite parity are closely located in energy. And this is what happens for $j_{15 / 2}, i_{11 / 2}$ and even $g_{9 / 2}$ in this mass region. Finally criterion (c) which was used in [3] as an evidence for near stable octupole deformation, has been rediscussed also by the same authors in [4] where they now admit that it is a necessary but not sufficient criterion.

We now discuss argument (d) concerning the lack of the so-called two-phonon octupole states at around double the energy of the one-phonon state. First, we would remind the reader that it may be somewhat hasardous to extrapolate from even nuclei to odd- $A$ nuclei in this transition region where the presence of one extra odd particle may polarize the even core in a considerable way. Further, we would also refer to our recent work [15] where we clearly show that the first non-rotational excited levels of ${ }^{222} \mathrm{Ra}$ and ${ }^{224} \mathrm{Ra}$ can be explained in terms of octupole vibrations exhibiting large anharmonicities. A proper treatment of these within the multiphonon method suggests that the first excited $0^{+}$states observed near $915 \mathrm{keV}$ in these nuclei may be mainly of an octupole nature. It shows also that there are no lower $0^{+}$states of this nature, explaining by the way the lack of the so-called two-phonon octupole states at low energy. This explanation obtained in a microscopic approach using a reflection symmetric basis must however not be used, per se, as an argument against stable octupole deformation. This could only be done if, in a similar calculation, a reflection asymmetric basis would appear to be less diagonal. To our knowledge, no such result is yet available. We would also like to add that the language used here may be somewhat misleading. Instead of speaking of vibrations exhibiting strong anharmonicities, one may perhaps rather refer to as finite amplitude collective motion described in a microscopic way.

In summary, we have analysed in all the details the various arguments used in favour of stable octupole deformation of the transitional nucleus ${ }^{225} \mathrm{Ra}$. Most of those appear to be necessary but not sufficient. Equal success and a good overall description of the low-lying bands in ${ }^{225} \mathrm{Ra}$ could be obtained either with or without reflection asymmetry. The actual results do not really tell anything about stable or dynamic octupole deformation. The only slight point in favour of stable asymmetry is the possibility to obtain a $1 / 2^{+}$as a ground state, but even this argument may be in some way included in the uncertainty of the single particle field. We do not claim that the description based on the reflection symmetry is superior to that based on stable octupole deformation, but merely that the latter is perhaps not the only solution for the description of transitional nuclei like ${ }^{225} \mathrm{Ra}$. 
On the one hand, further experimental evidence is needed to assert stable octupole deformation in this nucleus. On the other hand, microscopic calculations in odd- $A$ nuclei taking into account higher $K=0$ octupole excitations, which have been shown to be important in even mass nuclei, have to be undertaken.

\section{Acknowledgments.}

I would like to thank R. Chasman and Z. Szymanski for valuable comments and interesting discussions.

\section{References}

[1] Nybo, K. et al., Nucl. Phys. A 408 (1983) 127.

[2] Ragnarsson, I., Phys. Lett. B 130 (1983) 353.

[3] Sheline, R. K. et al., Phys. Lett. B 133 (1983) 13.

[4] Leander, G. A. and Sheline, R. K., Nucl. Phys. A 413 (1984) 375.

[5] Kurcewicz, W. et al., Nucl. Phys. A 270 (1976) 175 ; A 289 (1977) 1 ; A 304 (1978) 77 ; A 356 (1981) 15 ; A 383 (1982) 1.

[6] Zimmermann, R., Phys. Lett. B 113 (1982) 199.

[7] Leander, G. A. et al., Nucl. Phys. A 388 (1982) 452.

[8] Thomann, J. and Piepenbring, R., J. Physique 33 (1972) 613.

[9] Lamm, I. L., Nucl. Phys. A 125 (1969) 504.

[10] Chasman, R. R. et al., Rev. Mod. Phys. 49 (1977) 833.

[11] Chasman, R. R., Phys. Lett. B 96 (1980) 7.

[12] Rekstad, J. and Lovhøiden, G., Nucl. Phys. A 267 (1976) 40.

[13] Piepenbring, R. and Silvestre-Brac, B., in preparation.

[14] Monsonego, G. and Piepenbring, R., J. Physique 29 (1968) 42.

[15] Piepenbring, R., Phys. Rev. C 27 (1983) 2968. 